\title{
Bridging the Gap between Multimedia Applications and Heterogeneous Networks using a Market-based Middleware
}

\author{
Johan Kristiansson, Jeremiah Scholl, and Peter Parnes \\ Department of Computer Science \& Electrical Engineering, Division of Media Technology, \\ Luleå University of Technology, 97187 Luleå, Sweden \\ Email: \{Johan.Kristiansson, Jeremiah Scholl, Peter.Parnes\} @ltu.se
}

\begin{abstract}
This paper ${ }^{1}$ presents a middleware that applies a microeconomic model to help multimedia applications utilize available bandwidth in a way that maximizes the user's net benefit. The key components are a bandwidth broker that puts the supply of available bandwidth on a virtual market, and utility functions for each media, which are used to calculate the relative gain they can offer the user by using this bandwidth. Basic supply and demand principles are used where the broker raises the price if the total demand from all media exceeds the available supply, or lowers the price if the demand is lower than the available supply.
\end{abstract}

\section{Introduction}

Distributed multimedia applications provide users with a variety of inherently dynamic media, each having bandwidth requirements that can rapidly change over time. While a significant amount of research has targeted the creation of specific media that can adapt to bandwidth fluctuations (i.e. layered video coding), a relatively unexplored problem is how to share bandwidth efficiently between the different media inside an application in order to provide the user with the optimal aggregate experience.

Solving this problem in an optimal way requires a large amount of interdisciplinary knowledge. Designers must be able to handle an increasingly complex network infrastructure and handle issues such as IP mobility, congestion control, and bandwidth sharing in order to give an effective supply of bandwidth to an application, and deal with usability issues in order to map the relative gain that each media can give a user when allocated a portion of this bandwidth.

\footnotetext{
${ }^{1}$ This work was done within the VITAL project, which is supported by the Objective 1 Norra Norrland - EU structural fund programme for Norra Norrland. Support was also provided by the Centre for Distance-spanning Technology (CDT) and Mäkitalo Research Centre (MRC).
}

This paper presents a middleware framework based on micro-economical principals of supply and demand that deals with this issue. The middleware consists of a virtual marketplace that functions as a centralized point for the buying and selling of bandwidth. This allows the various solutions related to network management (usually effecting the supply) and the various solutions related to usability (usually effecting demand) to be researched and integrated separately into an application. Ultimately, this will serve to create better multimedia applications by allowing various experts from fields such as human computer interaction and computer communications to combine their knowledge in order to divide bandwidth between the different media in the way that provides the most benefit to the user.

\subsection{Related work}

A considerable amount of research has been carried out to provide QoS support in distributed multimedia systems $[3,5,6]$. However, existing QoS middlewares focus specifically on sharing resources between applications running on the same machine, or in the same network, rather than helping a multimedia application utilize the available bandwidth to it in the best possible way. This differs from the middleware presented in this paper, in that it uses microeconomic theory in a novel way by applying it inside applications in order to make it easy to combine various network services, such as IP-mobility and congestion control while maintaining the efficient use of resources and maximizing the benefit to the user.

\section{Market-based bandwidth management}

Microeconomic theory deals with production, purchase and sales of commodities that are in limited supply [1]. In the context of this framework, the commodity on the market is bandwidth, and is traded by two key players: consumers and suppliers. Consumers attempt to optimize their gain by 
purchasing commodities that give them the maximum gain at the lowest price, and suppliers try to sell commodities the highest price they can get in order to maximize their own profit. This leads to a variable pricing system that works like an "invisible hand" in order to distribute and allocate resources efficiently despite the selfish actions of each player. Eventually this price fluctuation will reach a state where the demand for goods at the going price equals the supply for goods at that price. When this state is reached, the market is said to be in equilibrium [1].

In practice equilibrium prices can be difficult to calculate because both demand and supply vary over time. The supply will for example vary depending on the type of connection currently used, congestion, or because of wireless interference. The demand may vary due to a wide variety of factors unique to every application. For example, the demand for the video stream of a particular user may vary during a video conference depending on communication patters such as who is the current speaker [7].

One way of handling these fluctuations is to use a tâtonnement process [1] in order to adjust the price iteratively until an equilibrium price is obtained. In this way, the seller decreases the price if their production is not sold and increases the price if the demand exceeds the supply.

$$
p_{n+1}(x)=p_{n}(x) \cdot \frac{d}{s}
$$

Equation (1) shows how the price is iteratively calculated based on supply and demand using a tâtonnement process, with $p_{n}(x)$ representing the current price, $p_{n+1}(x)$ next price, $d$ the aggregate demand of all media, and $s$ the current supply. Note that one advantage of this method is that specific knowledge about each individual consumer or suppliers is not required, which makes it possible to develop and integrate different components separately while allowing them to contribute to the equilibrium price once they have been added to the application.

\subsection{Overview of the middleware}

Figure 1, shows an overview of the proposed middleware, which operates on the market principles described above. The key player in the virtual marketplace is the Bandwidth Broker Agent (BBA), which acts as a go between in order to connect all the buyers and sellers on the market. Thus, the BBA sells bandwidth to all the different media streams used in the application, while obtaining bandwidth from various network management components. Note that the middleware does not require any support from the network infrastructure in form of QoS support or explicit charge models as the middleware is purely an endto-end solution.

The Bandwidth Consumer Agent (BCA) is responsible for purchasing bandwidth on behalf of a media. By using

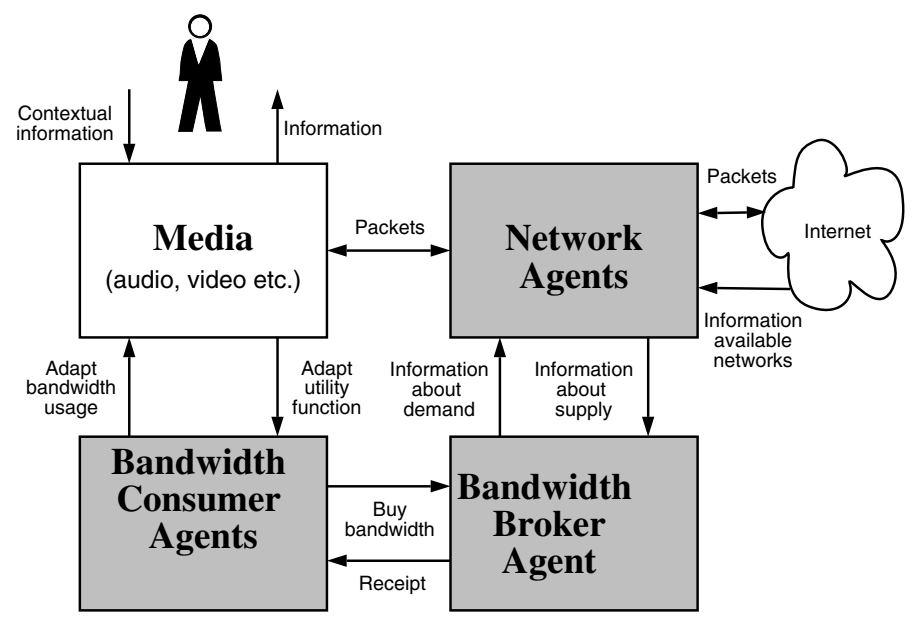

Figure 1. Overview of middleware.

an optimization method described in subsection 2.3, it calculates the total amount and type bandwidth that should be purchased in order to maximize the benefit to the user. The BCA also communicates information to the media it represents regarding how much bandwidth it has purchased for them, so that they can adapt based on the amount of bandwidth currently available to them.

The Network Agent (NA) contributes to the marketplace by obtaining the actual supply of bandwidth that will be sold by the BBA. It is also responsible for passing information about this available bandwidth to the BBA so that it can be sold to the various media. The NA can be fairly simple, or support a wide range of services depending on the components available to the application. For example, an NA with simple components added to it could calculate the supply using nothing more than a basic congestion control mechanism whereas a more advanced NA could handle network management functionality related to mobility management in order to obtain bandwidth from various networks.

\subsection{Calculating the supply}

The total supply that the broker can put onto the market is directly related to the amount of bandwidth available to the application and can be bounded by a variety of factors. Most often this supply will be equal to the perceived capacity of the network but for non-flat-rate connections it may also be bounded by budget controls set by the user.

Due to the varying requirements for individual applications and media, there is no one-size-fits-all way of calculating the available bandwidth. A large amount of research has been done over the past few years on how 
to calculate the bandwidth limit for non-TCP traffic and this has resulted in the creation of numerous protocols to serve applications with different characteristics. For unicast traffic the most notable of these is the TCP-Friendly Rate Control Protocol [2], which uses an equation based send rate in order to deliver TCP-friendly traffic at a smoother rate than is provided by TCP.

For multicast traffic the problem is a bit more complex and has thus resulted in the creation of a wider variety of protocols. In general, these protocols follow one of two strategies, either relying on the sender to adapt its send rate in a way that serves the needs of the entire receiver set (sender-based congestion control), or relying on the sender to make many quality levels available concurrently through separate channels, allowing each receiver to "sign up" for the appropriate channel(s) independently (receiverbased congestion control.

This means that for multicast traffic the type of bandwidth being supplied on the market will change somewhat depending on the underlying congestion control scheme in use. When using purely sender-based congestion control the broker supplies bandwidth for media that wish to send out packets on the network, whereas the broker supplies bandwidth for packets to be received when a receiver-based congestion control scheme is in use. Similarly, if a combination of sender-based and receiver-based congestion control is being used by the application then the broker will sell both bandwidth to be sent, and bandwidth to be received.

\subsection{Calculating the demand}

In order for a media to decide how much bandwidth to buy given the price $p$, it must calculate the relative gain the media can offer the user if allocated the bandwidth. This is done by creating utility functions for each media, where each utility function $u(x)$ maps the gain with different bandwidth levels. Concave utility functions are commonly used in classical microeconomic theory, and are useful in this context since they give a fairly accurate model of media that are less sensitive to bandwidth changes when allocation reaches some maximum requirement [4].

Video is a good example of a media that falls into this category since human beings can only notice a difference in the frame rate up until about 25 frames-per-second and tend to be more more sensitive to changes below 15 frames per second. For example, they tend to gain more when raising the frame rate from 1 to 6 frames per second than from 20 to 25 frames per second. Since each media wants to provide the user with the maximum net benefit (also known as the consumer surplus, $C S$ ) at a given price level, it can calculate the amount of bandwidth it should attempt to purchase by finding the maximum difference between the utility function and the price function.

If the utility function is concave, the $C S$ for media $m$ can be found by calculating the $x_{C S}^{m}$, where

$$
\frac{\delta\left(u\left(x_{C S}^{m}\right)\right)}{\delta x_{C S}^{m}}=\frac{\delta\left(p\left(x_{C S}^{m}\right)\right)}{\delta x_{C S}^{m}}
$$

The aggregate demand is used to calculate the new price during each iteration in equation 1 and is calculated as the summation of the results from each of the individual media.

Creating accurate utility curves for real world use may be a fair challenge, and therefore it is not expected that in most situations the user will be given this responsibility in any explicit way. However, application designers with a fair amount of expertise about the operation and use of their application should be able to create fairly robust utility curves that serve the general needs of users. One of the advantages of our framework is that it allows this work to be done by usability specialists, without requiring them to tackle complex issues related to network management, as those can be contained completely within the NA.

\section{References}

[1] C. Courcoubetis and R. Weber. Pricing Communication Networks;Economics, Technology and Modelling. Wiley, 2003.

[2] S. Floyd, M. Handley, J. Padhye, and J. Widmer. Equation-Based Congestion Control for Unicast Applications. In ACM SIGCOMM, pages 43-56, 2000.

[3] B. Li. Agilos: A Middleware Control Architecture for Application-Aware Quality of Service Adaptations. $\mathrm{PhD}$ thesis, University of Illinois, USA, 2000.

[4] Raymond R.F. Liao and A. T. Campbell. A Utility-Based Approach for Quantitative Adaptation in Wireless Packet Networks. Wireless Networks, 7(5):541-557, 2001.

[5] K. Nahrstedt, D. Xu, D. Wichadakul, and B. Li. QoSAware Middleware for Ubiquitous and Heterogeneous Environments. IEEE Communications Magazine, 39(11):140-148, 2001.

[6] R. Rajkumar, C. Lee, J. Lehoczky, and D. Siewiorek. A Resource Allocation Model for QoS Management. In Proceedings of the IEEE Real-Time Systems Symposium, 1997.

[7] J. Scholl, S. Elf, and P. Parnes. User-interest Driven Video Adaptation for Collaborative Workspace Applications. In International Workshop on Networked Group Communication NGC, pages 3-12, 2003. 